\title{
Production of QED pairs at small impact parameter in relativistic heavy ion collisions
}

\author{
Kai Hencken* \\ Institut für Physik, Universität Basel, 4056 Basel, Switzerland \\ Gerhard Baur ${ }^{\dagger}$ \\ Institut für Kernphysik, Forschungszentrum Jülich, Postfach 1913, 52425 Jülich, Germany \\ Dirk Trautmann \\ Institut für Physik, Universität Basel, 4056 Basel, Switzerland
}

(Received 18 February 2004; published 21 May 2004)

\begin{abstract}
The STAR Collaboration at the Relativistic Heavy Ion Collider is measuring the production of electronpositron pairs at small impact parameters, larger than but already close to the range, where the ions interact strongly with each other. We calculate the cross section relevant for the STAR experimental setup, as well as, differential distributions of the pair production process with the electromagnetic excitation of both ions in a semiclassical approach and within a lowest order QED calculation. We compare the distribution of electron and positron with the one coming from the cross section calculation without tagging for the excitation of the two ions. Finally we give an outlook of possible results at the LHC.
\end{abstract}

DOI: 10.1103/PhysRevC.69.054902

\section{INTRODUCTION}

Pair production in relativistic heavy ion collisions has attracted interest in the past mainly due to the fact that the strong fields allow for multiple pairs to be produced. At impact parameter of the order of twice the nuclear radius, but still larger than this, so that the two ions do not interact hadronically with each other (that is, the regime of the socalled "ultraperipheral collisions" UPC), the total pair production multiplicity is found to be about 1.5 for $\mathrm{Au}-\mathrm{Au}$ collisions at the Relativistic Heavy Ion Collider RHIC and about 3.9 for $\mathrm{Pb}-\mathrm{Pb}$ collisions at the LHC. These results are based on a lowest order QED calculation [1] and one might suspect that at these small impact parameters the strong fields of the two ions do lead to higher order corrections.

Therefore it is of interest to measure electron-positron pairs produced in such collisions and compare their distribution with theoretical predictions, e.g., in lowest order QED. The STAR Collaboration has recently measured $e^{+} e^{-}$pairs in collisions, which were selected by a trigger, looking for the simultaneous excitation of the two ions (mainly to the giant dipole resonance GDR) in addition to the pair production process [2-4], see Fig. 1. Such an event is characterized by the subsequent emission of one or only a few neutrons, which are then detected in the forward ZDC (zero degree calorimeter). This tagging was first proposed in order to study vector meson production [5-7]. Mutual excitation of the two ions is also used for the luminosity measurement at RHIC [8,9].

As the average impact parameter in such collisions is only about [10]

\footnotetext{
*Electronic address: k.hencken@unibas.ch; URL: http://quasar.unibas.ch/ hencken/

†Electronic address: g.baur@fz-juelich.de

*Electronic address: dirk.trautmann@unibas.ch
}

PACS number(s): 12.20. $-\mathrm{m}, 25.75 .-\mathrm{q}, 25.20 .-\mathrm{x}$

$$
\bar{b}=\frac{\int d^{2} b b P(b)}{\int d^{2} b P(b)} \approx \frac{8 R_{a}}{3} \approx 19 \mathrm{fm},
$$

one may expect strong field effects to be present for the pairs.

Due to the design of the STAR detector only electrons and positrons having a transverse momentum $p_{t}>65 \mathrm{MeV} / c$ and being emitted with a rapidity $|y|<1.15$ can be detected. As most of the pairs produced in ultraperipheral collisions (UPCs) are emitted with energies of the order of a few $m_{e} c^{2}$ and at small angles, such a measurement can only look at the tails of the distribution of the pairs. Even this momentum range was only possible using a lowered magnetic field in the STAR detector.

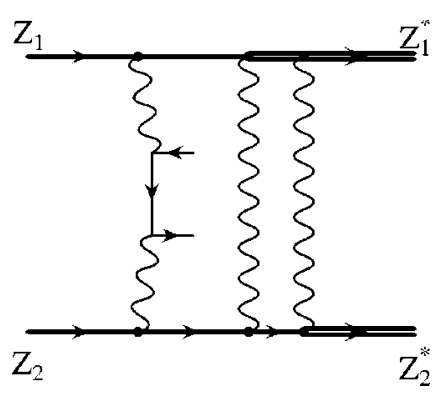

FIG. 1. The pair production together with the electromagnetic excitation of both ions, predominantly to the giant dipole resonance (GDR) is shown as one typical Feynman diagram. The process in lowest order involves at least the exchange of four photons (many more "soft Coulomb photons" are exchanged as well). Due to this the process predominantly occurs at small impact parameter, where the electromagnetic fields are strong, in contrast to the "untagged cross section" (without electromagnetic excitation of the ions), which has contributions coming also from large impact parameters. 
One approach to the calculation of this process is the use of the impact parameter dependent equivalent photon approximation [11-14]. The additional electromagnetic processes are easily incorporated in this semiclassical approach and the cross section can be expressed as

$$
\begin{aligned}
& \frac{d^{6} \sigma_{e^{+} e^{-}, 2 G D R}}{d^{3} p_{+} d^{3} p_{-}} \\
& \quad=\int d^{2} b P_{G D R}^{2}(b) \frac{d^{4} L}{d^{2} b d \omega_{1} d \omega_{2}} \frac{d^{6} \sigma_{\gamma \gamma \rightarrow e^{+}, e^{-}}\left(\omega_{1}, \omega_{2}\right)}{d^{3} p_{+} d^{3} p_{-}},
\end{aligned}
$$

where $\sigma_{\gamma \gamma \rightarrow e^{+}, e^{-}}$denotes the cross section for real photons and $d^{4} L / d^{2} b d \omega_{1} d \omega_{2}$ the impact parameter dependent photonphoton luminosity (for the details of the photon-photon luminosity, see Sec. 3 of Ref. [15] and Sec. 2.7 of Ref. [16] and references therein). One difficulty in this approach is the correct choice of the cutoff parameter present in the expression for the photon spectrum. This is especially difficult due to the smallness of $m_{e}$, which is much smaller than the "usual cutoff" imposed on the maximal transverse momentum of the photon from the elastic form factor of the ion, which is given by $1 / R_{A} \approx 80 \mathrm{MeV}$. For a discussion see Ref. [16], especially Chap. 7, beginning of p. 412. It was found that the total cross section for electron-positron pair production (given by a full QED calculation in lowest order [17] or alternatively by the approximate analytic expression of Racah [18], which coincide within the numerical accuracy of the calculations) is only reproduced with a cutoff chosen around $m_{e}$. On the other hand it was also found that neither a choice of $m_{e}$ nor of $R_{A}$ is able to reproduce the total impact parameter dependent probability $P(b)$ at impact parameter smaller than the Compton wavelength $\lambda_{c}=386 \mathrm{fm}$ from an exact QED calculation [1]. For a discussion about the choice of the cutoff parameter see, e.g., Ref. [19]. In addition, in the usual semiclassical approach the transverse momentum distribution of the photons is integrated over. In order to get, for example, the transverse momentum distribution of the pair, one needs to take this momentum distribution into account to get the correct final result. For a possible approach taking this into account from first principles see Refs. [20,21]. Due to this it is of interest to do an exact calculation of this process in lowest order QED in order to compare with experimental results, as well as, in order to understand whether the equivalent photon approximation is a valid approximation in this case.

In Sec. II we show how our calculation is done in lowest order QED and in the semiclassical approximation. This is then used in Sec. III to calculate cross sections and differential distributions for RHIC and also for possible LHC conditions. The comparison with the experimental results has been done in the meantime and will be presented by the STAR Collaboration in another publication [22].

\section{CALCULATION OF PAIR PRODUCTION AND NUCLEAR EXCITATION IN LOWEST ORDER QED}

The STAR experiment at RHIC measures the pair production cross section together with the double electromagnetic excitation in both ions, see Fig. 1. In order to incorporate the experimental conditions in the theoretical calculation, it is most appropriate to work in the semiclassical approach. Using the fact, that in this approach the probabilities of the individual processes factorize and are given by the product of the individual probabilities (for a theoretical description of this approach, see Ref. [10]) we can write the cross section for this process as

$$
\frac{d^{6} \sigma_{e^{+} e^{-}, 2 G D R}}{d^{3} p_{+} d^{3} p_{-}}=2 \pi \int_{b_{\text {min }}}^{\infty} b d b P_{G D R}^{2}(b) \frac{d^{6} P(b)}{d^{3} p_{+} d^{3} p_{-}} .
$$

The minimum impact parameter was chosen to be $b_{\min }$ $=2 R_{a} \approx 14 \mathrm{fm}$, where we assume the nuclei to touch, that is, interact hadronically with each other. The use of the semiclassical description in this case is not only justified due to the strong Coulomb interaction between the two ions (leading to a large number of "soft" Coulomb photons exchanged between them), but also simplifies the calculation of this higher order process (tagged process: pair production plus two GDR excitation processes) considerably.

Following Ref. [23] the probability for GDR excitation in one ion is to a good approximation given as

$$
P_{G D R}(b)=S / b^{2},
$$

with

$$
S=\frac{2 \alpha^{2} Z^{3} N}{A m_{N} \omega} \approx 5.45 \times 10^{-5} Z^{3} N A^{-2 / 3} \mathrm{fm}^{2},
$$

where $m_{N}$ denotes the nucleon mass, and the neutron, proton, and mass number of the ions are $N, Z$, and $A$, respectively (we consider only symmetric collisions here, the calculation can trivially be extended to incorporate also asymmetric systems). The excitation probability is inversely proportional to the energy $\omega\left(\approx 80 \mathrm{MeV}^{-1 / 3}\right)$ of the GDR state. Neutrons are not only emitted from the GDR excitation but are also coming from higher excited states $[8,24,25]$. These can be taken into account approximately by increasing $S$ accordingly. As this does not change the $1 / b^{2}$ behavior for the small impact parameter, we are interested in, and only leads to a rescaling of the cross section, not the form of the differential distributions, we have used in our calculation the more simple value of $S$ in Eq. (5). Of course one needs then to include in addition the different decay channels into one, two, etc., neutrons [26-28], as well as, the fact that the probability for GDR excitation is already quite large (about 0.5 for $b_{\text {min }}$ ) so that multiphoton excitation mechanisms need to be included as well. Assuming a Poisson distribution for the different (independent) excitation processes, one would need to replace $P_{G D R}(b)$ then with [24]

$$
P_{A \rightarrow A^{*}}(b)=1-\exp \left[P_{G D R}(b)\right] .
$$

One sees that the multiphoton excitation tends to reduce again the probability (it has to stay below the unitarity limit of 1). In the Appendix we show how a more complex $b$ dependence than a simple $1 / b^{2}$ for $P_{G D R}(b)$ can be calculated 

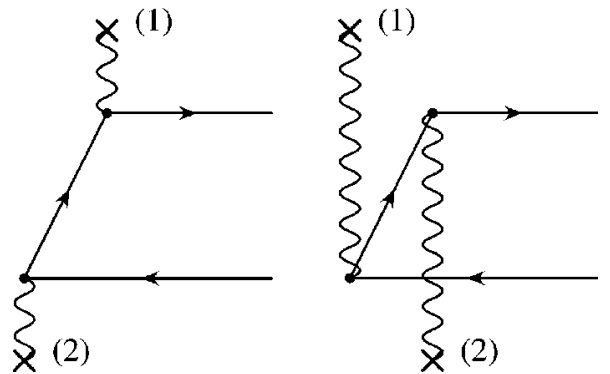

FIG. 2. The two Feynman diagrams contributing to pair production in lowest order QED are shown. The crosses denote the coupling to the (external) Coulomb field of one of the ions.

within the same approach. Therefore an improved calculation can be done in this way. On the other hand for the current experimental accuracy the simplified approach seems to be appropriate.

With this the cross section for pair production together with the GDR excitation of both ions is given by

$$
\begin{aligned}
\frac{d^{6} \sigma_{e^{+} e^{-}, 2 G D R}}{d^{3} p_{+} d^{3} p_{-}}= & 2 \pi \int_{b_{\min }}^{\infty} b d b P_{G D R}^{2}(b) 2 \pi \\
& \times \int q d q \frac{d^{6} \hat{P}(q)}{d^{3} p_{+} d^{3} p_{-}} J_{0}(q b) \\
= & (2 \pi)^{2} S^{2} \int q d q \frac{d^{6} \hat{P}(q)}{d^{3} p_{+} d^{3} p_{-}} \int_{b_{\min }}^{\infty} b d b \frac{J_{0}(q b)}{b^{4}} \\
= & (2 \pi)^{2} S^{2} \int q d q \frac{d^{6} \hat{P}(q)}{d^{3} p_{+} d^{3} p_{-}} \int_{b_{\min }}^{\infty} \frac{d b}{b^{3}} J_{0}(q b),
\end{aligned}
$$

where we have introduced the two-dimensional Fourier transform of the impact parameter dependent probability for pair production $d^{6} P(b) / d^{3} p_{+} d^{3} p_{-}$as

$$
\begin{aligned}
\frac{d^{6} P(b)}{d^{3} p_{+} d^{3} p_{-}} & =\int d^{2} q \exp (i \vec{q} \vec{b}) \frac{d^{6} \hat{P}(\vec{q})}{d^{3} p_{+} d^{3} p-} \\
& =2 \pi \int q d q \frac{d^{6} \hat{P}(q)}{d^{3} p_{+} d^{3} p_{-}} J_{0}(q b) .
\end{aligned}
$$

We rewrite the integral over $b$ in dimensionless units as

$$
\int_{b_{\text {min }}}^{\infty} \frac{d b}{b^{3}} J_{0}(q b)=q^{2} \int_{q b_{\text {min }}}^{\infty} \frac{d x}{x^{3}} J_{0}(x)=: q^{2} I_{3}\left(q b_{\text {min }}\right) .
$$

Following the derivation of Refs. [1,17], one can calculate the two-dimensional Fourier transform of $d^{6} P(\vec{b}) / d^{3} p_{+} d^{3} p_{-}$ in lowest order QED. The two Feynman diagrams for this process in the semiclassical approximation are shown in Fig. 2. One gets the differential probability as

$$
\begin{aligned}
& \frac{d^{6} \hat{P}(q)}{d^{3} p_{+} d^{3} p_{-}}=(Z \alpha)^{4} \frac{4}{\beta^{2} \gamma^{4}} \frac{1}{(2 \pi)^{6} 2 \epsilon_{+} 2 \epsilon_{-}} \int d^{2} q_{1}\left[N_{0} N_{1} N_{3} N_{4}\right]^{-1} \\
& \times \operatorname{Tr}\left\{( p _ { - } + m ) \left[N_{2 D}^{-1} \boldsymbol{u}^{(1)}\left(p_{-}-\boldsymbol{q}_{1}+m\right) \boldsymbol{u}^{(2)}+N_{2 X}^{-1} \boldsymbol{u}^{(2)}\right.\right. \\
& \left.\times\left(\boldsymbol{q}_{1}-\not p_{+}+m\right) \boldsymbol{u}^{(1)}\right]\left(p_{+}-m\right)\left[N _ { 5 D } ^ { - 1 } \boldsymbol { u } ^ { ( 2 ) } \left(p_{-}-\boldsymbol{d}_{1}^{\prime}\right.\right. \\
& \left.\left.+m) \boldsymbol{u}^{(1)}+N_{5 X}^{-1} \boldsymbol{u}^{(1)}\left(\boldsymbol{q}_{1}^{\prime}-\not p_{+}+m\right) \boldsymbol{u}^{(2)}\right]\right\} \text {, }
\end{aligned}
$$

with

$$
\begin{gathered}
N_{0}=-q_{1}^{2}, \quad N_{1}=-\left[q_{1}^{2}-\left(p_{+}+p_{-}\right)\right]^{2}, \\
N_{3}=-\left(q_{1}+q\right)^{2}, \quad N_{4}=-\left[q_{1}+\left(q-p_{+}-p_{-}\right)\right]^{2}, \\
N_{2 D}=-\left(q_{1}-p_{-}\right)^{2}+m^{2}, \quad N_{2 X}=-\left(q_{1}-p_{+}\right)^{2}+m^{2}, \\
N_{5 D}=-\left[q_{1}+\left(q-p_{-}\right)\right]^{2}+m^{2}, \\
N_{5 X}=-\left[q_{1}+\left(q-p_{+}\right)\right]^{2}+m^{2},
\end{gathered}
$$

with the longitudinal components of $q_{1}$ given by $q_{10}=\frac{1}{2}\left[\left(\epsilon_{+}\right.\right.$ $\left.\left.+\epsilon_{-}\right)+\beta\left(p_{+z}+p_{-z}\right)\right], \quad q_{1 z}=[1 / 2 \beta]\left[\left(\epsilon_{+}+\epsilon_{-}\right)+\beta\left(p_{+z}+p_{-z}\right)\right]$ $=(1 / \beta) q_{10}$, and $u^{(1,2)}=\gamma(1,0,0, \pm \beta)$ are the four velocities of the two ions, $\gamma$ and $\beta$ are the Lorentz factor and velocity of each ion in the center-of-mass frame. We have included in addition a nuclear form factor $F(q)$. We choose for ease of computation in our case a monopole form factor of the form

$$
F(q)=\frac{\Lambda^{2}}{\Lambda^{2}-q^{2}}=\frac{\Lambda^{2}}{\Lambda^{2}+Q^{2}},
$$

where $\Lambda^{2}=6 /\left\langle R^{2}\right\rangle$ is set to about $80 \mathrm{MeV}$ in order to reproduce the rms radius of the ion. This leads in the terms $N_{0}^{-1}$, $N_{1}^{-1}, N_{3}^{-1}$, and $N_{4}^{-1}$ to a replacement of the term $1 / q^{2}$ by $F(q) / q^{2}$. The integration over $d^{2} q_{1}$ can be done analytically, using the usual tricks for Feynman integrations in two dimensions. For details of this, we refer the reader to Ref. [1].

The integral $I_{3}\left(z=q b_{\min }\right)$ in Eq. (9) can be solved analytically and calculated easily, as is shown in the Appendix.

Finally we make the integral over $d q$ dimensionless to get

$$
\frac{d^{6} \sigma_{e^{+} e^{-}, 2 G D R}}{d^{3} p_{+} d^{3} p_{-}}=(2 \pi)^{2} \frac{S^{2}}{b_{\text {min }}^{4}} \int z^{3} d z \frac{d^{6} \hat{P}\left(\frac{z}{b_{\text {min }}}\right)}{d^{3} p_{+} d^{3} p_{-}} I(z) .
$$

For the "untagged differential cross section" (that is without triggering on the additional electromagnetic excitations of the ions), we integrate over $\vec{b}$ without the factor $P_{G D R}^{2}(b)$ in Eq. (3). As the contribution coming from $b<b_{\min }$ is small in this case, we have extended (only here) the integration over all $b$. 


$$
\begin{aligned}
\frac{d^{6} \sigma_{e^{+} e^{-}}}{d^{3} p_{+} d^{3} p_{-}} & =\int d^{2} b \frac{d^{6} P(b)}{d^{3} p_{+} d^{3} p_{-}} \\
& =\int d^{2} b d^{2} q \frac{d^{6} \hat{P}(q)}{d^{3} p_{+} d^{3} p_{-}} \exp (i \vec{q} \vec{b}) \\
& =(2 \pi)^{2} \int d^{2} q \delta(\vec{q}) \frac{d^{6} \hat{P}(q)}{d^{3} p_{+} d^{3} p_{-}} \\
& =(2 \pi)^{2} \frac{d^{6} \hat{P}(0)}{d^{3} p_{+} d^{3} p_{-}} .
\end{aligned}
$$

This approach was pursued in Ref. [17] and differential and integrated cross sections were calculated. For the untagged cross sections we do not take a nuclear form factor of the two ions into account, as pair productions occurs predominantly at large impact parameter and for small $q^{2}$ of the two photons.

Whereas for the untagged cross section only the value of $\hat{P}(q)$ for $q=0$ is needed, here our expression, Eq. (12), is a folding over a range of $q$ given in terms of $1 / b_{\min }$. In order to compare the differential distributions in both the tagged and in the untagged case we have also made calculations of the untagged cross section with the same kinematical restrictions as in the case with GDR excitations.

The expression of Eq. (12) is in a form, which can be evaluated using a Monte Carlo integration for both the integration over $z$, as well as, the six-dimensional integration over $p_{+}$and $p_{-}$at the same time. For this we have used VEGAS [29]. Both $P(q)$ and $J_{0}^{\text {int }}$ are oscillatory functions, having both positive and negative values, which could lead to cancellations. Looking at $z^{3} I(z)$ together with the result of $P\left(z / b_{\text {min }}\right)$ one sees that the integrand falls off for large $z$, that is, for large $q$. It is found that the main contribution comes from the region around $z=2$ and that the contribution from the negative part at larger $z$ are suppressed. The integration will have positive and negative contributions but the cancellations between them are not severe. With the help of VEGAS we can get the integrated cross section and also differential cross sections by binning the differential results.

\section{RESULTS}

We have made calculations of the cross section and of differential distributions of the electron, the positron and the pair including the experimental restrictions at STAR. The integration over $b$ (or equivalently $q$ ) is incorporated into the Monte Carlo integration. Another strategy would be to calculate $d^{6} \hat{P}(q) / d^{3} p_{+} d^{3} p_{-}$for different values of $q$ and fixed values of $p_{+}, p_{-}$and do a Bessel transform in each case. For the integrated cross section, that is, $\hat{P}(q)$, this can be done and was done as an independent check of our approach. To obtain differential cross sections, this approach is rather cumbersome.

In a first step, we have calculated the total cross section (with the kinematical conditions of the STAR experiment) as a function of $b_{\text {min }}$, the minimum impact parameter, by using three different approaches: We can calculate $P(b)$ directly for

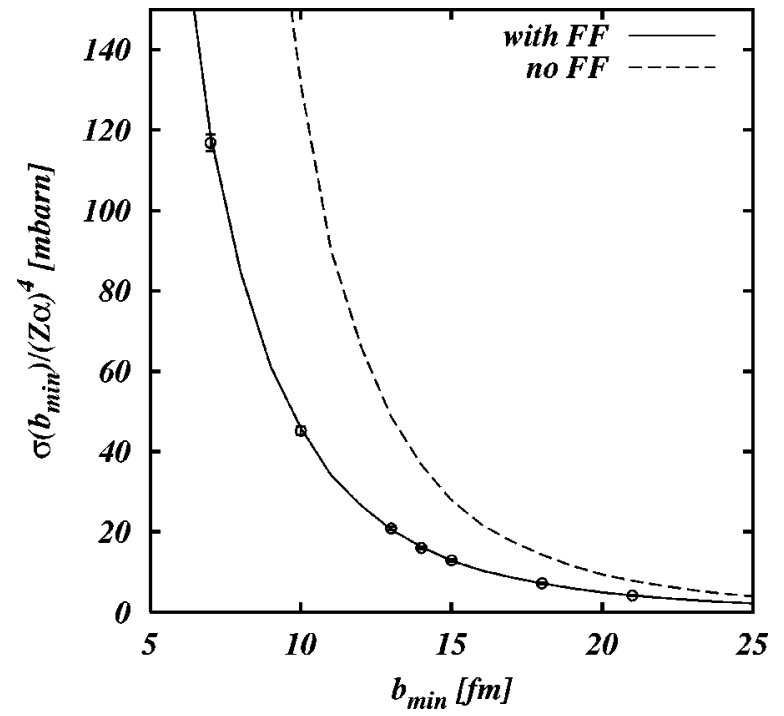

FIG. 3. The tagged cross section including kinematical restrictions of the STAR experiment are shown as a function of the minimal allowed impact parameter $b_{\min }$. Calculations with and without a form factor for the nucleus are shown. The lines were calculated by calculating $P(b)$ first and integrating then over $b$. These lines are in perfect agreement with a similar approach, where $P(q)$ is calculated first and then integrated over $q$. The circles correspond to the results of our Monte Carlo approach, where, as explained in the text, both the integration over $q$ and over $p_{+}$and $p_{-}$are done within the Monte Carlo integration routine.

the pair production process and integrate numerically over $b$. Second we can start from $\hat{P}(q)$ directly and do the integration over $q$ numerically via the Fourier transformed of $P_{G D R}(b)$, that is, using $I(z)$. Finally we have done the calculation with the integration over $q$, that is, $z$, directly with the Monte Carlo integration. In all three cases we have restricted the phase-space integration over the momenta of electron and positron according to the experimental conditions of STAR: $p_{t}>60 \mathrm{MeV} / c$ and $|y|<1.15$ for each lepton. The results are shown in Fig 3. The lines correspond to calculations with and without a monopole form factor for the nucleus, showing that the incorporation of a form factor is important. All three approaches agree quite well with each other, showing that our approach is working well.

From this we get an integrated cross section for $\mathrm{Au}-\mathrm{Au}$ collisions at RHIC, including the restrictions $\left|p_{t}\right|>60 \mathrm{MeV} / c,|y|<1.15$ of $2.30,1.76,1.43 \mathrm{mb}$, for $b_{\text {min }}$ $=13,14$, and $15 \mathrm{fm}$, respectively. In addition we have calculated a number of differential distributions, which were also studied at STAR. The cross section as a function of the transverse momenta and energy distribution of the electron and positron are shown in Fig. 4. In lowest order QED the distribution of electron and positron are identical to each other. The difference between the two distributions can therefore be seen as a measure of the accuracy the Monte Carlo integration. One can look also at properties of the produced pair: The transverse momentum and the invariant mass of the pair are shown in Fig. 5. We have not shown the rapidity distribution, which we found to be more or less flat over the allowed range. In all four diagrams we show also the differ- 

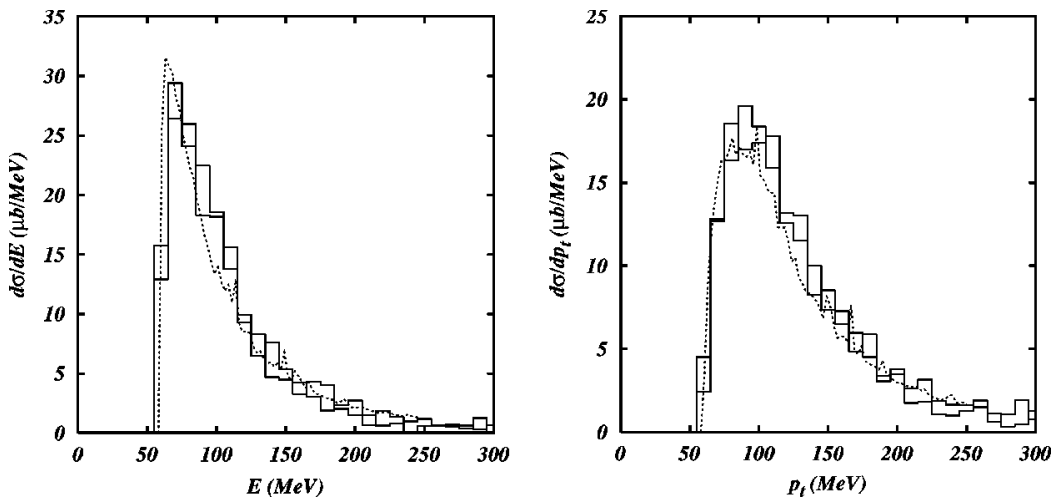

FIG. 4. Energy and transverse momentum distributions are shown for the electron and the positron, corresponding to the STAR experimental conditions. In lowest order QED the two distributions are identical and the spread between the two is a measure of the uncertainties coming from the Monte Carlo integration. This is compared with an untagged cross section calculation (dotted line), divided by a factor 183 to give the same integrated cross section.

ential distributions of the untagged cross section. The total untagged cross section (within the STAR acceptance range) was found to be $0.322 \mathrm{~b}$. We have rescaled therefore the untagged cross section by a factor of 183 , so that the integrated cross section is the same as in the tagged case and their shape can be better compared. We see that the transverse momentum distribution and the energy distribution of the individual leptons are more or less identical in shape. The same is also true for the invariant mass distribution, with the only exception that the "tail" at low invariant masses is higher for the untagged distributions. The biggest effect is seen in the transverse momentum distribution of the pair. Here we also expect the effect of the small impact parameter (corresponding to larger transverse momenta of the photons) to be largest. The slower fall-off at larger transverse momenta is most probably due to the fact that no form factor was used in the calculation of this cross section, which should be visible at $P_{t}>80 \mathrm{MeV} / c$. In order to investigate the effect of the nuclear form factor and in order to understand the large difference between the cross section with and without nuclear form factor, see Fig. 3 above, we show in this plot also the transverse momentum distribution of the pair for a calculation without form factor. It can be seen that in this case the cross section gets sizeable contributions for $P_{t}>80 \mathrm{MeV} / c$.

We have studied in addition the question, whether especially the distribution as a function of $P_{t}$ of the pair is sensitive to the form of $P_{G D R}(b)$ used in our approach. Using a more general approach, see Appendix for details, one expect that the next correction is of the form $P_{G D R}^{3}(b) \sim 1 / b^{6}$ instead of $P_{G D R}^{2}(b) \sim 1 / b^{4}$. In such a model the average impact parameter changes from about $8 R_{a} / 3 \approx 19 \mathrm{fm}$ to about
$12 R_{a} / 5 \approx 17 \mathrm{fm}$, which is a small change compared to the Compton wavelength of the electron $(400 \mathrm{fm})$, but is still a $10 \%$ reduction of this average impact parameter. We have therefore studied the distribution of electron and positron under this condition. As expected the differential distributions are found to be the same within the uncertainties of the Monte Carlo approach used.

As an outlook for future experiments we are showing results and distributions one might expect to see for $\mathrm{Pb}-\mathrm{Pb}$ collisions at the LHC. Using the same kinematical restrictions as for the STAR experiment, the results are shown in Figs. 6 and 7. The integrated cross section is found to be $2.90 \mathrm{mb}$ for the tagged case.

Finally as a rather optimistic estimate we have calculated also the differential cross section for a kinematical range of $\left|p_{t}\right|>2.6 \mathrm{MeV} / c$ and $|y|<1.5$, where ALICE will be able to detect the electrons with its internal trigger system (ITS), even though it will not be able to measure energies or momenta.

With these kinematical conditions we can study the question, whether ALICE will be able to see multiple pairs produced in a single collisions. For this we calculate the impact parameter dependent probability under the kinematical conditions. One finds that for impact parameters close to $b_{\min } P(b) \approx 20 \%$. Following Refs. [16,17,30,31] we use a Poisson distribution as a good approximation for the $N$ pair production probability

$$
P(N, b)=\frac{P(b)^{N}}{N !} \exp [-P(b)] .
$$

Multiplying with $P_{G D R}^{2}(b)$ and integrating over $b$ we get the cross section for $1,2, \ldots, N$ pair production.
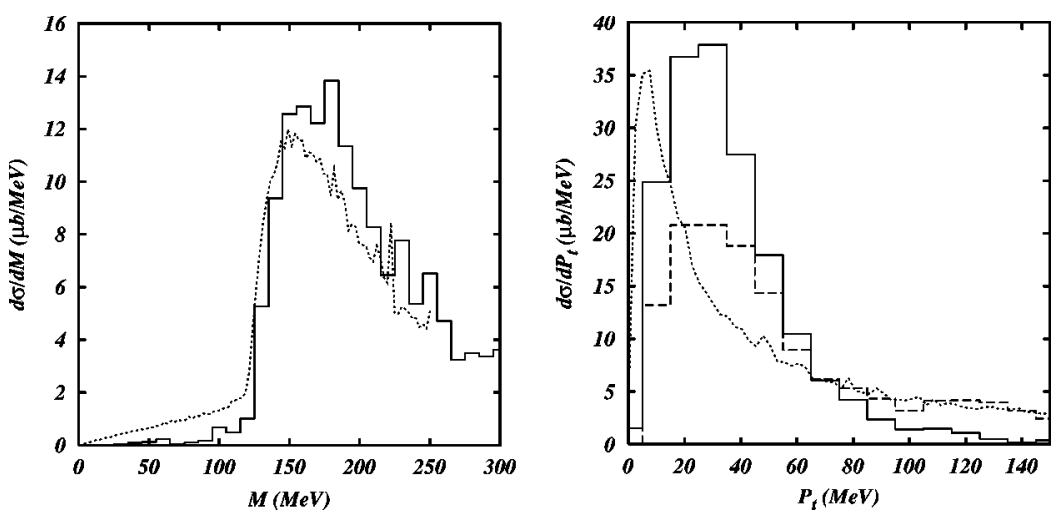

FIG. 5. The cross section of the $e^{+} e^{-}$pair as a function of the invariant mass and the transverse momentum are shown. Again the results are compared with the untagged cross section (dotted line). In addition we have plotted the result of the tagged calculation without form factor (dashed line). Both the untagged cross section and the one without form factor have been normalized (by dividing them with a factor 183 and 2.74, respectively) to give the same integrated cross section as the tagged case including the form factor. 

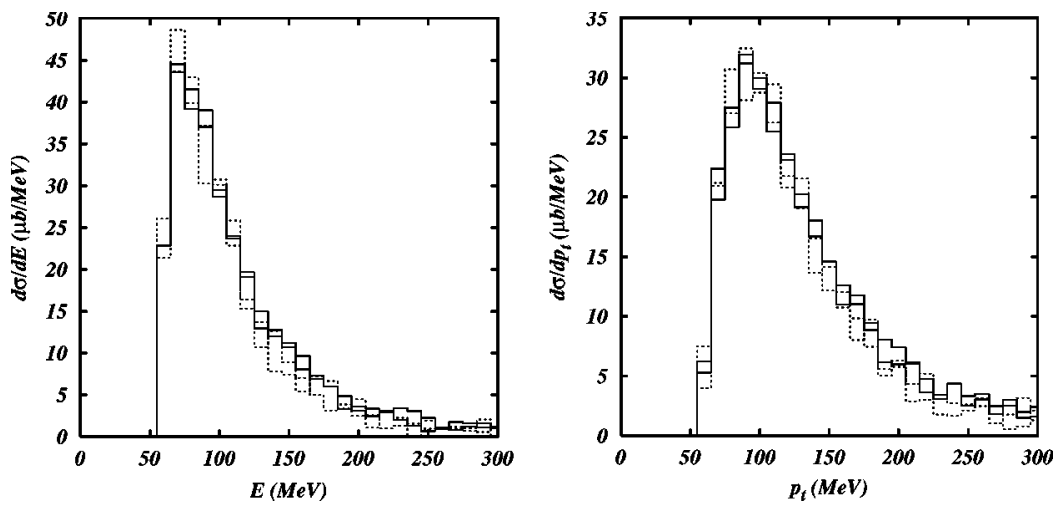

FIG. 6. Energy and transverse momentum distribution are shown for the two leptons for $\mathrm{Pb}-\mathrm{Pb}$ collisions at the LHC (solid line). This is compared with the rescaled spectrum (factor 1.65) of $\mathrm{Au}-\mathrm{Au}$ collisions at RHIC (dotted line).

$$
\sigma(N)=\int d^{2} b P_{G D R}^{2}(b) P(N, b)
$$

The result as a function of $b_{\min }$ is shown in Fig. 8 together with the total integrated cross section

$$
\sigma_{\text {total }}=\sum_{N=1}^{\infty} \sigma(N)
$$

The cross section one would get from the Born cross section can be interpreted as a "multiplicity" cross section

$$
\sigma_{\text {Born }}=\int d^{2} b P_{G D R}^{2}(b) P(b)=\sum_{N=1}^{\infty} N \sigma(N),
$$

and would be relevant in order to calculate the number of pairs produced (in contrast to the number of events). One can see that about $10 \%$ of all events are multiple pair production events and accordingly also about $10 \%$ of all pairs are produced in a multiple pair production process. This shows that at ALICE one should be able to detect and study multiple pair production.

We have investigated a similar question also for RHIC using as an estimate for a possible range $p_{t}>50 \mathrm{MeV} / c$ and $2.5<y<4$.0. Unfortunately the probability for pair production under these conditions is only of the order of a few permille and therefore the multiple pair production cross section is less than one permille of the single pair production cross section, making such an investigation difficult.

\section{DISCUSSION AND OUTLOOK}

We have calculated cross section and differential distributions of the pair production process in ultraperipheral heavy ion collisions in lowest order QED for the simultaneous electromagnetic excitation of both ions. We have seen that the most sensitive quantity is the transverse momentum distribution, which differs the most from the distribution of the untagged process. As the comparison will show [2,22] our results were found to be in good agreement with the experimental results. On the other hand only about 50 events were found at STAR, so the overall statistics is not very good. Additional runs might give better statistics. Still our analysis shows that the data at the moment give no sign that higher order Coulomb effects are large for pairs produced with these large transverse momenta [32]. Such higher order Coulomb effects would lead most likely to an asymmetry of the electron and positron distribution especially for the transverse momentum distribution. At the moment however no calculation exists, which describes consistently the effects of the strong Coulomb fields of both pairs on the pair production process at small impact parameter. Due to the experimental conditions of RHIC, the probability for pair production is well below one; the multiple pair productions effects are therefore rather small.

As already mentioned above the transverse momentum cut of $p_{t}>65 \mathrm{MeV} / c$ at STAR was only possible due to a reduced magnetic field. There are currently plans to use even lower magnetic fields and also making use of other detectors within STAR [33] in order to extend the measurements both to smaller transverse momenta and to larger rapidities. It remains to be seen, whether the new phenomenon of mul-
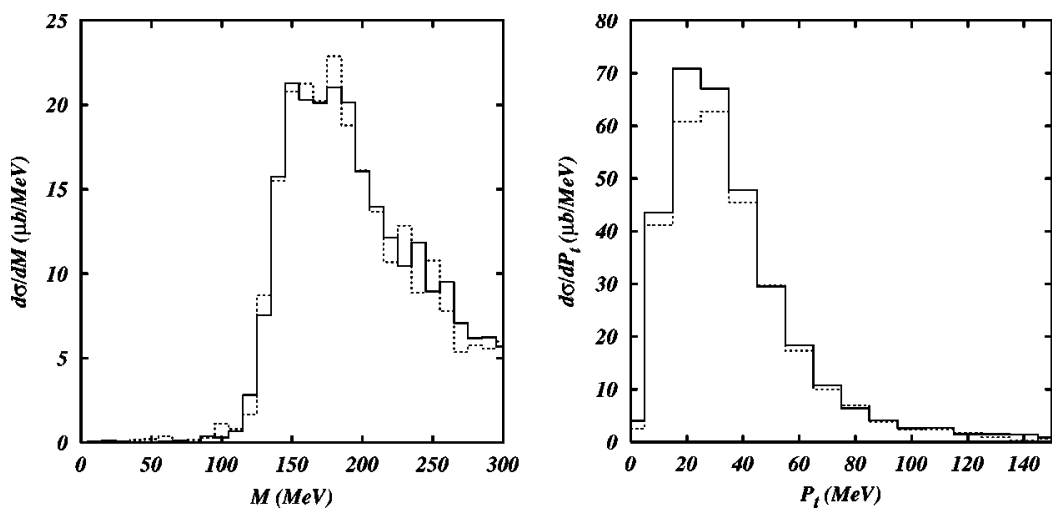

FIG. 7. The invariant mass and the transverse momentum of the $e^{+} e^{-}$pair is shown for $\mathrm{Pb}-\mathrm{Pb}$ collisions at the LHC (solid line). The results are compared with the rescaled spectrum (factor 1.65) of Au-Au collisions at RHIC (dotted line). 


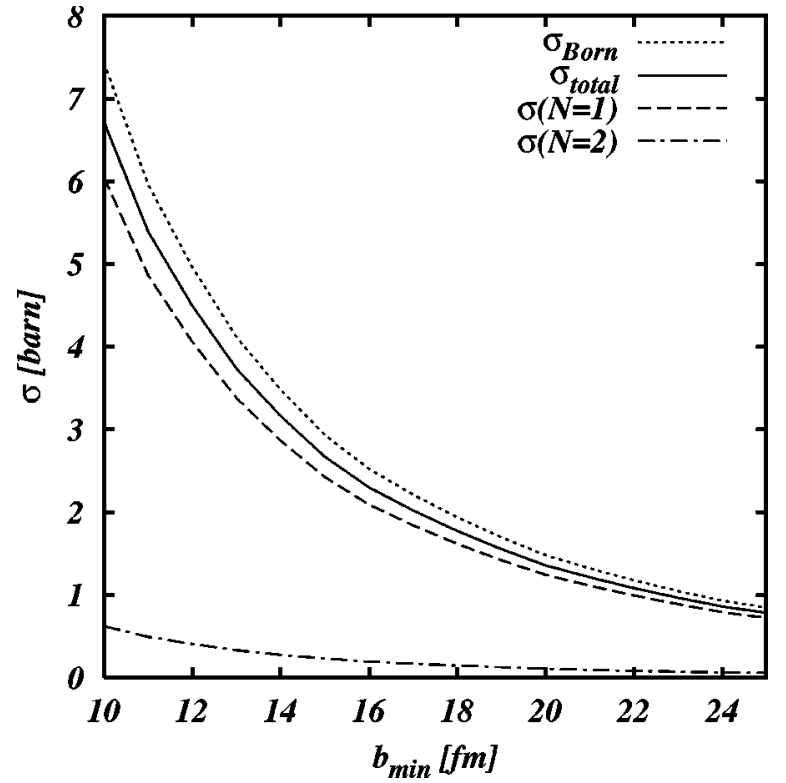

FIG. 8. The different cross sections for single and multiple pair production together with the mutual electromagnetic excitation of both ions are shown for the kinematical conditions at ALICE. The cross section is shown as a function of $b_{\text {min }}$, the minimal impact parameter. See the text for further details of the different cross sections.

tiple pair production will then be detected. On the other hand with the low transverse momentum cutoff of the ITS at ALICE/LHC, about $10 \%$ of all pair production events are going to be multiple pair production processes, therefore one should expect that this new phenomena will be observed easily there. The fact that no kinematical information and also no particle identification is possible at ALICE, will make such a measurement still a challenge.

\section{ACKNOWLEDGMENTS}

The authors would like to thank Spencer R. Klein and Vladimir B. Morozov for interesting discussions and collaborations on this subject and (S.K.) for critical reading of the manuscript. Discussions with Serguei Sadovsky and Yuri Kharlov have been important in understanding the possibilities of detecting the pair production at LHC.

\section{APPENDIX}

Throughout our calculations we have assumed that only the GDR excitation is relevant for the triggering and therefore a simplified dependence on $b$, see Eq. (4), has been used. In this appendix we want to show that this is not a real limitation, but that other impact parameter dependencies can be treated as well. For example, assuming that the higher resonant states of the GDR are excited through a Poisson process, we would need to replace $P_{G D R}(b)$ by $1-\exp \left[-P_{G D R}(b)\right]$. In general we assume that the relevant $P_{A \rightarrow A^{*} \rightarrow X+x n}(b)$ can be expressed as a series of inverse powers of $b$

$$
P_{A \rightarrow A^{*} \rightarrow X+x n}(b)=\sum_{n=0}^{\infty} \frac{S_{n}}{b^{n}} .
$$

Using this in the expression for $d^{6} \sigma_{e^{+} e^{-}, 2 G D R} /\left(d^{3} p_{+} d^{3} p_{-}\right)$, see Eq. (12), we need to calculate generalizations of $I(z)$, Eq. (9), of the form

$$
I_{n}(z):=\int_{z}^{\infty} \frac{d x}{x^{n}} J_{0}(x)
$$

with $I_{3}(z)$ corresponding to the one used in our calculations.

For the calculation of these integrals, we first use the following recursion relation:

$$
I_{n}(z)=\frac{J_{0}(z)}{(n-1) z^{n-1}}-\frac{J_{1}(z)}{(n-1)^{2} z^{n-2}}-\frac{1}{(n-1)^{2}} I_{n-2}(z) .
$$

This relation can be easily derived by partial integration and by using the well-known recursion relations between the Bessel functions $J_{n}(z)$ [34]. By repeated application of Eq. (A3) every $I_{n}$ with odd and even $n$ can be reduced to the starting values $I_{1}(z)$ and $I_{0}(z)$, respectively, which are given in the literature [34]:

$$
I_{0}(z)=1-z_{1} F_{2}\left(\frac{1}{2} ; 1, \frac{3}{2} ;-\frac{z^{2}}{4}\right)
$$

and

$$
I_{1}(z)=\frac{z^{2}}{8}{ }_{2} F_{3}\left(1,1 ; 2,2,2 ;-\frac{z^{2}}{4}\right)-\ln \frac{z}{2}-\gamma,
$$

where $\gamma$ is the Euler constant.

These two expressions can be calculated easily by the rapidly converging power series of the hypergeometric functions or by using suited polynomial expressions given in the literature [34]. We are here only interested in the case, where $n=2 m+1$ is an odd number, in which case the complete recursion relation is given by

$$
\begin{aligned}
I_{2 m+1}(z)= & \frac{(-1)^{m}}{m !^{2} 2^{2 m+1}}\left\{J_{0}(z) \sum_{s=1}^{m} s !(s-1) !\left(-\frac{4}{z^{2}}\right)^{s}\right. \\
& \left.-\frac{z}{2} J_{1}(z) \sum_{s=1}^{m}(s-1) !^{2}\left(-\frac{4}{z^{2}}\right)^{s}+I_{1}(z)\right\} .
\end{aligned}
$$

Moreover this equation can be further simplified, by splitting off the terms singular at $z=0$ (the principal part of the Laurent expansion in $z$ ), by using the power series for the Bessel functions:

$$
J_{m}(z)=\left(\frac{z}{2}\right)^{m} \sum_{k=0}^{\infty} \frac{\left(-z^{2} / 4\right)^{k}}{k !(k+m) !}
$$

and by rearranging the resulting sum. After some straightforward algebra we thus obtain the compact expression for $m$ $\geqslant 0$ 


$$
I_{2 m+1}(z)=\frac{(-1)^{m}}{2^{2 m+3}(m+1) !^{2}} z_{2}^{2} F_{3}\left(1,1 ; 2,2+m, 2+m ;-\frac{z^{2}}{4}\right)
$$$$
-\frac{(-1)^{m}}{2^{2 m}(m) !^{2}}\left(\ln \frac{z}{2}+\gamma\right)+\sum_{k=0}^{m} a_{m, k^{2}} z^{-2 k}
$$

where

$$
a_{m, 0}=\frac{(-1)^{m}}{2^{2 m} m !^{2}} \sum_{s=1}^{m} \frac{1}{s}, \quad a_{m, k}=\frac{(-1)^{m-k}}{2^{2(m-k)+1}(m-k) !^{2} k} k \geqslant 1 .
$$

For $m=1$ we get the explicit expression

$$
\begin{aligned}
& { }_{2} F_{3}\left(1,1 ; 2,2+m, 2+m ;-\frac{z^{2}}{4}\right) \\
& \quad=(m+1) !^{2}\left(-\frac{4}{z^{2}}\right) \sum_{k=1}^{\infty} \frac{\left(-\frac{z^{2}}{4}\right)^{k}}{k(k+m) !^{2}} .
\end{aligned}
$$

We use this rapidly converging power series in our numerical calculations. For the case where $n$ is an even number, the same approach can be used. For completeness we only give here the final result:

$$
\begin{aligned}
I_{2 m}(z)= & \frac{1}{z^{2 m-1}(2 m-1)}{ }_{1} F_{2}\left(-m+\frac{1}{2} ;-m+\frac{3}{2}, 1 ;-\frac{z^{2}}{4}\right) \\
& +\frac{(-1)^{m} 2^{2 m} m !^{2}}{(2 m) !^{2}} .
\end{aligned}
$$

[1] K. Hencken, D. Trautmann, and G. Baur, Phys. Rev. A 51, 1874 (1995).

[2] F. Meissner and V. B. Morozov, presented at "Small-x and Diffraction 2003", Sept. 17-20, 2003, Fermilab, nucl-ex/ 0307006.

[3] V. B. Morozov, Ph.D. thesis, University of California, 2003.

[4] S. Klein, nucl-ex/0310020.

[5] A. J. Baltz, S. R. Klein, and J. Nystrand, Phys. Rev. Lett. 89, 012301 (2002).

[6] S. Klein and J. Nystrand, Phys. Rev. Lett. 84, 2330 (2000).

[7] S. Klein and J. Nystrand, Phys. Rev. C 60, 014903 (1999).

[8] A. Baltz, C. Chasman, and S. N. White, Nucl. Instrum. Methods Phys. Res. A 417, 1 (1998).

[9] M. Chiu et al., Phys. Rev. Lett. 89, 012302 (2002).

[10] G. Baur et al., Nucl. Phys. A729, 787 (2003).

[11] G. Baur, in International Workshop on Relativistic Aspects of Nuclear Physics, Rio de Janeiro, Brazil 1989, edited by T. Kodama et al. (World Scientific, Singapore, 1990), p. 127.

[12] G. Baur and L. G. Ferreira Filho, Nucl. Phys. A518, 786 (1990).

[13] M. Greiner, M. Vidović, J. Rau, and G. Soff, J. Phys. G 17, L45 (1991).

[14] N. Cahn and J. D. Jackson, Phys. Rev. D 42, 3690 (1990).

[15] G. Baur, K. Hencken, and D. Trautmann, J. Phys. G 24, 1657 (1998).
[16] G. Baur et al., Phys. Rep. 364, 359 (2002).

[17] A. Alscher, K. Hencken, D. Trautmann, and G. Baur, Phys. Rev. A 55, 396 (1997).

[18] G. Racah, Nuovo Cimento 14, 93 (1937).

[19] V. M. Budnev, I. F. Ginzburg, G. V. Meledin, and V. G. Serbo, Phys. Rep., Phys. Lett. 15, 181 (1975).

[20] M. Vidović, M. Greiner, C. Best, and G. Soff, Phys. Rev. C 47, 2308 (1993).

[21] G. Baur and N. Baron, Nucl. Phys. A561, 628 (1993).

[22] J. Adams et al., nucl-ex/0404012.

[23] C. A. Bertulani and G. Baur, Phys. Rep. 163, 299 (1988).

[24] A. J. Baltz, M. J. Rhoades-Brown, and J. Weneser, Phys. Rev. E 54, 4233 (1996).

[25] A. J. Baltz and M. Strikman, Phys. Rev. D 57, 548 (1998).

[26] I. A. Pshenichnov et al., Phys. Rev. C 64, 024903 (2001).

[27] I. A. Pshenichnov et al., Phys. Rev. C 60, 044901 (1999).

[28] I. A. Pshenichnov et al., Phys. Rev. C 57, 1920 (1998).

[29] G. P. Lepage, J. Comput. Phys. 27, 192 (1978).

[30] K. Hencken, D. Trautmann, and G. Baur, Phys. Rev. A 51, 998 (1995).

[31] M. C. Güçlü et al., Phys. Rev. A 51, 1836 (1995).

[32] S. J. Brodsky and J. Gillespie, Phys. Rev. 173, 1011 (1968).

[33] S. Klein (private communication).

[34] M. Abramowitz and I. A. Stegun, Handbook of Mathematical Functions (Dover, New York, 1965). 